\title{
A Wideband Direct Data Domain Genetic Algorithm Beamforming
}

\author{
Hassan ELKAMCHOUCHI, Mohamed HASSAN \\ Dept. of Electrical Engineering, University of Alexandria, Alhuria Street, Alexandria, Egypt \\ helkamchouchi@hotmail.com, engmohamedmokhtar1@yahoo.com
}

\begin{abstract}
In this paper, a wideband direct data-domain genetic algorithm beamforming is presented. Received wideband signals are decomposed to a set of narrow subbands using fast Fourier transform. Each sub-band is transformed to a reference frequency using the steering vector transformation. So, narrowband approaches could be used for any of these sub-bands. Hence, the direct datadomain genetic algorithm beamforming can be used to form a single 'hybrid' beam pattern with sufficiently deep nulls in order to separate and reconstruct frequency components of the signal of interest efficiently. The proposed approach avoids most of drawbacks of already-existing statistical and gradient-based approaches since formation of a covariance matrix is not needed, and a genetic algorithm is used to solve the beamforming problem.
\end{abstract}

\section{Keywords}

Wideband array, direct data-domain, genetic algorithm, signal of interest, matrix pencil method

\section{Introduction}

An adaptive array is able to electronically steer its main lobe to any desired direction and place deep pattern nulls to directions of interference sources. That way, the antenna could adaptively minimize the interference power while maintaining the array gain in the direction of the target signal [1-3].

Statistical methods of adaptive antennas are computationally intensive processes and require stationary data to construct a covariance matrix. Direct data-domain $\left(\mathrm{D}^{3}\right)$ methods could overcome drawbacks of statistical techniques by processing data on snapshot-by-snapshot basis without constructing a covariance matrix. Hence, $D^{3}$ methods could handle non-stationary environments and coherent interferers [4].

Genetic algorithms (GAs) might be more efficient than gradient-based methods for nulling a linear antenna array since the gradient-based methods have following disadvantages:

- The methods are highly sensitive to starting points when the number of variables, and hence the size of the solution space, increases.
- The methods frequently converge to local suboptimum solutions.

- The methods require a continuous and differentiable objective function.

- The methods require piecewise linear cost approximation (for linear programming).

- The methods have problems with convergence and algorithm complexity (for non-linear programming).

Future wireless systems have to utilize wideband smart antennas to meet high speed data transmission while avoiding undesired interference [15]. Beamforming techniques used in narrowband systems are inappropriate for wideband systems due to the limited ability of tracking a desired user or forming nulls in directions of interfering sources over a large frequency band [5], [6]. Some earlier work has been done to solve such problems. Existing concepts of wideband beamforming exhibit disadvantages which could be summarized as follows:

- Different array patterns are used for different frequencies [12-14]. Obviously, such an approach is quite cumbersome.

- Gradient-based wideband beamforming [1], [7], [16] has the already described disadvantages.

- Statistical methods depend on the formation of a covariance matrix [17]. Therefore, stationary data are expected to estimate the covariance matrix. In case of non-stationary data, resulting errors in the covariance matrix reduce the ability to handle coherent interferers [4].

- In [7] it is mentioned that "although the main beam is directed to the signal of interest (SOI) direction and the jammers are nulled correctly but the nulls are not deep enough"; same drawback appears in [1]. Such disadvantage affects the accuracy of SOIs reconstruction. In addition, in [1] and [7], nulls' depths and DOAs estimations' accuracy are found to be frequency dependent. Therefore, selecting one beam pattern corresponding to single sub-band does not assure sufficient nulls' depths to cancel interferers in all other sub-bands.

The proposed wideband direct data-domain genetic algorithm ( $\mathrm{WD}^{3} \mathrm{GA}$ ) beamforming relies on decomposing the received wideband signals into a set of narrow sub- 
bands by using fast Fourier transform (FFT) and transform all sub-bands to a reference frequency by using the steering vector transformation [5]. Any of these sub-bands could be used by narrowband techniques for direction of arrival (DOA) estimation and beamforming. The investigated narrowband direct data-domain adaptive nulls genetic algorithm ( $\mathrm{D}^{3} \mathrm{ANGA}$ ) beamformer [9] is used to cancel jammers' frequency components in each sub-band. Finally, inverse fast Fourier transform could be used to retrieve the SOI in time domain.

$\mathrm{WD}^{3} \mathrm{GA}$ beamforming has the following unique set of characteristics:

- $\mathrm{WD}^{3} \mathrm{GA}$ beamforming uses only a single hybrid array beam pattern; thus the method complexity is reduced.

- GA is used to solve the beamforming problem. Hence, the gradient-based methods' drawbacks are avoided.

- Both FFT and covariance-matrix-based techniques require recorded samples of the received signals [4]. However, WD ${ }^{3} \mathrm{GA}$ beamforming doesn't make any assumption about the statistics of the environment. Therefore, data non-stationarity has a little effect on the method performance.

- The problem of frequency dependent estimations of DOAs and nulls' depths [1], [7] could be solved by taking into consideration that in $\mathrm{D}^{3} \mathrm{ANGA}$ beamforming [9], the determination of the nulls' depths and the DOAs estimation are done before and independent on the beamforming algorithm. Hence, for wideband beamforming, the nulls' depths could be selected prior to the beamforming algorithm to be proportional to strongest interferers' frequency components and the most accurate estimated DOAs could be selected priory. Hence, one 'hybrid' reconstruction array beam pattern combines both sufficient deep nulls and accurate estimated DOAs could be formed.

\section{Steering Vector Transformation}

For a wideband antenna array of $N$ elements and $d$ spacing between adjacent elements, consider $q+1$ uniformly spaced directions covering a pre-specified angular azimuth region $\Phi_{\mathrm{q}}[5]$, [7] where,

$$
\boldsymbol{\Phi}_{\mathrm{q}}=\left[\phi_{0}, \phi_{1}, \ldots, \phi_{q}\right]
$$

In this paper, all coming signals are considered to be in the azimuth plane $\left(\theta=90^{\circ}\right)$. The steering vector transformation is based on transforming the array steering matrix at the $k^{\text {th }}$ frequency $f_{k}$ to another array steering matrix at a pre-specified reference frequency $f_{o}$ using a transformation matrix $\boldsymbol{T}_{\mathrm{q}}(k)$ for the angular region $\Phi_{\mathrm{q}}$ such that:

$$
\boldsymbol{A}\left(\boldsymbol{\Phi}_{\mathrm{q}}, f_{o}\right)=\boldsymbol{T}_{\mathrm{q}}(k) \boldsymbol{A}\left(\boldsymbol{\Phi}_{\mathrm{q}}, f_{k}\right)
$$

where $\boldsymbol{A}\left(\boldsymbol{\Phi}_{\mathrm{q}}, f_{o}\right)$ and $\boldsymbol{A}\left(\boldsymbol{\Phi}_{\mathrm{q}}, f_{k}\right)$ are the array steering matrices for the angular region $\boldsymbol{\Phi}_{\mathrm{q}}$ at the $k^{\text {th }}$ frequency $f_{k}$ and at the reference frequency $f_{o}$ respectively. The array steering matrix could be computed as follows:

$$
\boldsymbol{A}\left(\boldsymbol{\Phi}_{\mathrm{q}}, f\right)=\left[\boldsymbol{a}\left(\phi_{0}, f\right), \boldsymbol{a}\left(\phi_{1}, f\right), \ldots, \boldsymbol{a}\left(\phi_{q}, f\right)\right]
$$

where $\boldsymbol{a}(\phi, f)$ is the steering vector defined by:

$$
\boldsymbol{a}(\phi, f)=\left[1, e^{\frac{2 \pi f d}{c} \cos (\phi)}, \ldots, e^{\frac{2 \pi f(N-1) d}{c} \cos (\phi)}\right]^{T}
$$

where $T$ denotes the transpose of the vector. Equation (3) could be solved for $\boldsymbol{T}_{\mathrm{q}}(k)$ using the least squares method which yields the solution:

$$
\boldsymbol{T}_{\mathrm{q}}(k)=\boldsymbol{A}\left(\Phi_{\mathrm{q}}, f_{o}\right) \boldsymbol{A}\left(\Phi_{\mathrm{q}}, f_{k}\right)^{H}\left(\boldsymbol{A}\left(\Phi_{\mathrm{q}}, f_{k}\right) \boldsymbol{A}\left(\Phi_{\mathrm{q}}, f_{k}\right)^{H}\right)^{-1}
$$

where the $H$ superscript represents the conjugate transpose of a complex matrix. The processed input voltage vector at the $k^{\text {th }}$ frequency which has been transformed to the reference frequency $f_{o}, \boldsymbol{x}\left(f_{o}\right)$, could be written as:

$$
\boldsymbol{x}\left(f_{o}\right)=\boldsymbol{T}_{\mathrm{q}}(k) \boldsymbol{x}\left(f_{k}\right)
$$

where $\boldsymbol{x}\left(f_{k}\right)$ is the input voltage vector at the $k^{\text {th }}$ frequency. Using this transformation, a single narrowband beamformer tuned at the reference frequency $f_{o}$ could be used.

\section{Matrix Pencil Method}

The MP method is a narrowband $\mathrm{D}^{3}$ method to estimate the DOA of various signals impinging on an antenna array; the signals' complex amplitudes could be estimated as well [4], [8].

For a uniformly linear array composed of $N+1$ element, the voltage induced in the array $n^{\text {th }}$ element, $x_{n}$, could be written as:

$$
x_{n}=\sum_{k=1}^{P} S_{k} e^{\left(\frac{j 2 \pi n d \cos \left(\phi_{k}\right)}{\lambda}\right)}+\xi_{n}=\sum_{k=1}^{P} S_{k} a_{k}^{n}+\xi_{n}
$$

where $\xi_{n}$ is the noise at the $n^{\text {th }}$ array element, $P$ is the number of incident signals, $S_{k}$ is the complex amplitude of the $k^{\text {th }}$ incident signal, $\lambda$ is the wave length, $d$ is the distance between two adjacent elements, $\phi_{k}$ is the DOA of the $k^{\text {th }}$ signal, and $a_{k}$ are the poles to be estimated.

The poles $a_{k}$ could be estimated by constructing and processing a Hankel matrix as illustrated in [4], [8], then the DOAs of various signals could be obtained as follows:

$$
\phi_{k}=\cos ^{-1}\left[\frac{\lambda \ln \left(a_{e s k}\right)}{j 2 \pi d}\right]
$$

where $a_{\mathrm{es} k}$ is the $k^{\text {th }}$ estimated pole. The complex amplitudes vector of the $P$ signals, $\boldsymbol{A M P}$, could be obtained by: 


$$
\boldsymbol{A M P}=\left(\boldsymbol{P}_{0}^{H} \boldsymbol{P}_{0}\right)^{-1} \boldsymbol{P}_{0}^{H} \boldsymbol{x}
$$

where $\boldsymbol{P}_{\mathbf{0}}$ is the matrix containing the pole of each incident signal at each antenna element and $\boldsymbol{x}$ is a vector containing the induced voltages at the array elements.

\section{4. $D^{3}$ ANGA Beamforming}

$\mathrm{D}^{3} \mathrm{ANGA}$ is a narrowband beamformer [9]. Consider a linear antenna array with uniformly spaced $N$ elements. Hence, $N$ complex weights are used for beamforming. Given the DOAs and the strengths of all coming signals, a genetic algorithm (GA) is used to find the optimal values of these weights in order to fulfill the algorithm objectives [10], [11] which are as follows:

- Minimizing beam the pattern average value to minimize the pattern side lobes level.

- Maximizing the pattern value in the direction of the SOI $\left(P_{S}\right)$ to radiate maximum possible power in this direction.

- Placing deep nulls in directions of the interferers. In addition, nulls' depths are selected to be proportional to interference incident signals' intensities.

Hence, the fitness function could be written as:

$$
\text { Fit }=w \sum_{i=1}^{i=J}|| \frac{P_{i}}{P_{s}}\left|-N_{i}\right|+\left|\frac{P_{a v}}{P_{s}}\right|
$$

where $J$ is the number of the interferer (jammer) signals, $P_{i}$ is the array beam pattern complex value in the direction of the $i^{\text {th }}$ jammer, $P_{S}$ is the array beam pattern complex value in the direction of the SOI, $N_{i}$ is the $i^{\text {th }}$ normalized pattern null value corresponding to the $i^{\text {th }}$ jammer, $w$ is the weighting factor used to balance GA optimization between the two terms of the fitness function. || denotes the absolute (magnitude) of the complex quantities and $P_{a v}$ is the pattern average value which could be calculated by:

$$
P_{a v}=\frac{\sum_{\theta} \sum_{\phi}|P(\theta, \phi)|}{N_{P}}
$$

where $N_{P}$ is the number of points at which the pattern values are calculated. The pattern value at any direction, $P(\theta, \phi)$, could be computed by

$$
P(\theta, \phi)=\boldsymbol{W}^{\mathrm{T}} \boldsymbol{A}(\theta, \phi)
$$

where $\boldsymbol{W}$ is the complex weights' vector to be estimated by the GA and $A(\theta, \phi)$ is the steering vector in the direction of $(\theta, \phi)$. Assuming all coming signals are in the azimuth plane $\left(\theta=90^{\circ}\right), A(\theta, \phi)$ could be expressed as:

$$
\boldsymbol{A}=\left[1, e^{j 2 \pi \frac{d}{\lambda} \cos \phi}, e^{j 2 \pi \frac{2 d}{\lambda} \cos \phi}, \ldots, e^{j 2 \pi \frac{(N-1) d}{\lambda} \cos \phi}\right]^{\mathrm{T}}
$$

where $d$ is the space between array adjacent elements and $\lambda$ is the wavelength corresponding to the operating frequency. The $i^{\text {th }}$ normalized pattern null value, $N_{i}$, corresponding to the $i^{\text {th }}$ jammer could be computed by:

$$
N_{i}=\left(\left|S_{i}\right| * C\right)^{-1}
$$

where $S_{i}$ is the $i^{\text {th }}$ jammer intensity and $C$ is the cancelling factor (CF) used to make the nulls' depths sufficient to cancel interference signals efficiently.

In this paper, all the pattern values and all the obtained complex weights are normalized with respect to $P_{s}$. The GA-estimated complex weights could be used to separate and reconstruct the SOI using:

$$
R S O I=\boldsymbol{W}_{n}^{T} \boldsymbol{x}
$$

where $R S O I$ is the reconstructed SOI, $\boldsymbol{W}_{n}$ is the normalized weights vector, and $\boldsymbol{x}$ is the received signals' vector.

\section{Genetic Algorithm Components}

GA is a powerful optimization technique based on the concept of natural selection and natural genetics [10]. GA repeatedly modifies a population of individual solutions. At each step the GA selects individuals at random from the current population to be parents and uses them to produce the children of the next population. Over successive generations, the population "evolves" toward an optimal solution which is considered to be the solution which gives the minimum of the fitness function [10], [11]. In this paper, GAs are implemented based on the built-in genetic algorithm of R2013a MATLAB software package. The basic GA components, used in this paper, are reviewed briefly as follows [10]:

- Genetic representation of solution: Real number encoding is used to represent individual solutions or chromosomes.

- Population initialization: uniform random initialization is used and the population size is selected to be ten times the number of the antenna array elements taking into consideration that two chromosomes are used to represent each array element complex weight one for the real part and the other for the imaginary part [11].

- Evaluation of the fitness function: The GA should find the global minimum of the fitness function.

- Fitness scaling: The ranking method in which the scaling of raw scores is based on the rank of each individual instead of its score is used [11].

- Selection methods: Stochastic uniform selection method is used. Stochastic uniform selection method lays out a line in which each parent corresponds to a section of the line of length proportional to its scaled value. The algorithm moves along the line in steps of equal size. At each step, the algorithm allocates a parent from the section it lands on. The first 
step is a uniform random number less than the step size. Also, Elitism forces GA to retain some number of the best individuals at each generation [10], [11]. In this paper, the most fit two chromosomes survive directly to the next generation as elite chromosomes.

- Genetic operators: genetic operators are used to produce new individuals. Crossover and mutation are the most frequently used genetic operators and are described as follows:

1- The crossover operator is the exchange of genes between parent's chromosomes to produce offspring. The scattered crossover method is used. In this method, crossover is done by creating a random binary vector and selecting the genes where the vector's elements are ones from the first parent, and the genes where the vector's elements are zeros from the second parent, and combines the genes to form the child [10], [11]. The fraction of each population, other than elite children, that are made up of crossover children is set to 0.8 . The remaining chromosomes are mutation children.

2- Mutation is done by the addition of a random number which is chosen from a Gaussian distribution to each entry of the parent vector.

- Termination condition: a maximum number of 500 generations is used to terminate GA.

\section{The Proposed WD ${ }^{3}$ GA}

$\mathrm{WD}^{3} \mathrm{GA}$ beamformer is implemented by a narrowband decomposition structure whereby each signal received at each array element is transformed into its frequency domain components using FFT. Each narrow frequency sub-band is transformed to a reference frequency using the steering vector transformation. The transformed sub-bands could be processed by narrowband techniques. Hence, they are sent to the MP stage, as shown in Fig. 1. In the MP stage, the DOAs as well as the complex magnitudes of all incident signals' frequency components are estimated.

One array beam pattern is used for reconstructing all SOI frequency components from all sub-bands. Two parameters determine the accuracy of reconstruction of the SOI frequency components: 1- the DOA estimation accuracy of all coming signals; 2- the pre-specified pattern nulls' depths. The estimated DOAs should be as accurate as possible, so it is recommended to select the DOAs estimated using the nearest sub-band to the reference frequency $f_{o}$ since it is observed that the nearest sub-band to the reference frequency has the minimum error in the steering vector transformation [5]. The error in the steering vector transformation could be calculated as follows:

$$
\text { Error }=\boldsymbol{A}\left(\boldsymbol{\Phi}_{\mathrm{q}}, f_{o}\right)-\boldsymbol{T}_{\mathrm{q}}(k) \boldsymbol{A}\left(\boldsymbol{\Phi}_{\mathrm{q}}, f_{k}\right) .
$$

When the reference frequency is selected to be one of the considered signals' frequencies, the steering vector transformation is not required for the corresponding subband. Hence, the corresponding steering vector transformation error is zero. For the array beam pattern nulls' depths, it is recommended to select the deepest nulls corresponding to the strongest estimated interferers' frequency components by the MP method in order to cancel all the interferers' frequency components in all sub-bands efficiently. So, one hybrid reconstruction beam pattern combines both accurate estimated DOAs and deep nulls and could be used for the estimation of all SOI frequency components then inverse FFT could be used to retrieve the SOI in time domain.

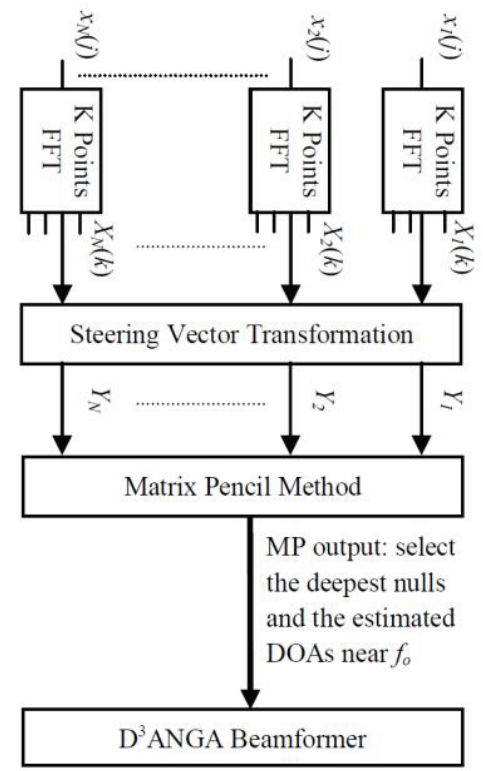

Fig. 1. The proposed $\mathrm{WD}^{3} \mathrm{GA}$ beamforming structure.

\section{Numerical Examples}

Consider a uniform linear array of 6 elements. The received wideband signals are decomposed into their narrowband components at 6 frequencies within the design frequency band [ $3 \mathrm{GHz}-4 \mathrm{GHz}$, these frequencies are: 3 , 3.2, 3.4, 3.6, 3.8, and $4 \mathrm{GHz}$. The inter-element spacing used equals half wavelength corresponding to the maximum frequency within the design frequency band in order to avoid spatial aliasing [5], [6]. Consider one SOI and one jammer, with arrival angles of $\phi=120^{\circ}$ and $\phi=100^{\circ}$ respectively. All SOI frequency components' amplitudes are equal to $1 \mathrm{~V} / \mathrm{m}$. The signal to noise ratio is $30 \mathrm{~dB}$ and the reference frequency is chosen to be $4 \mathrm{GHz}$. This configuration and values are used in the following two examples; the difference is in the jammer strength.

\subsection{Example 1: Constant Jammers Frequency Components Magnitudes}

In the $1^{\text {st }}$ example, all jammer frequency components' magnitudes are $40 \mathrm{~dB}$ over the corresponding SOI frequency components. Fig. 2 and Fig. 3 show the estimated 


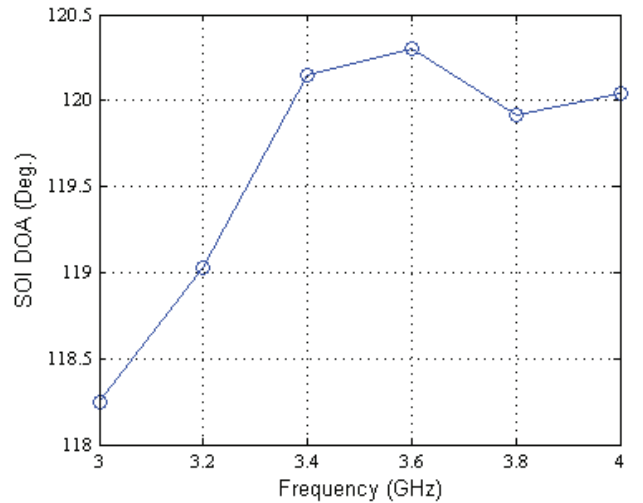

Fig. 2. SOI estimated DOA.

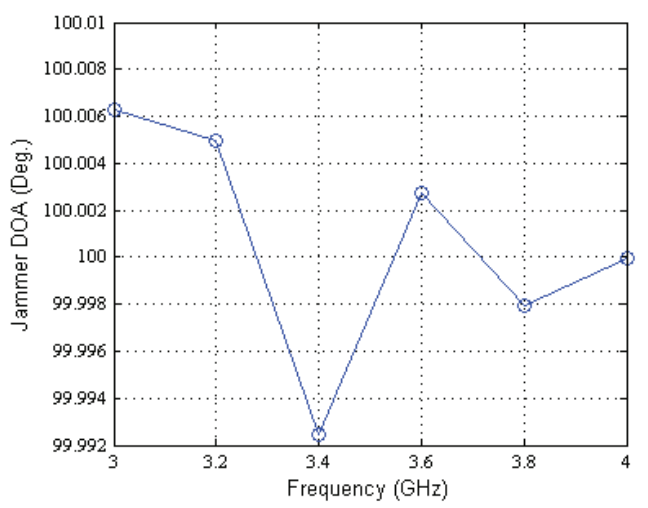

Fig. 3. Jammer estimated DOA.

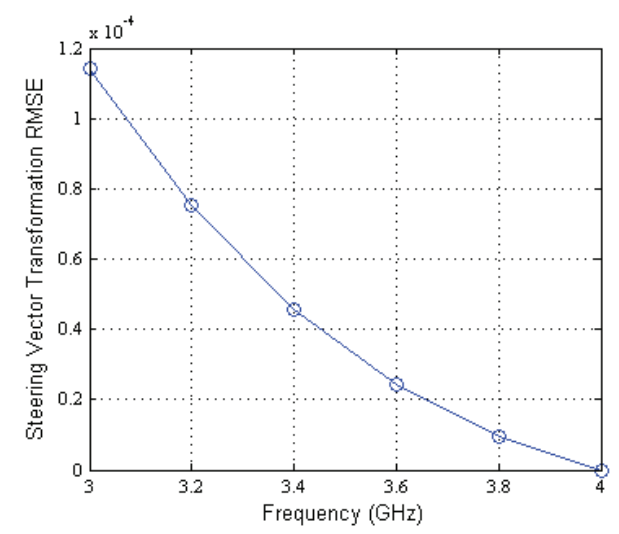

Fig. 4. Steering vector transformation RMSE.

DOA for both the SOI and the jammer signal, respectively, corresponding to each frequency sub-band. Obviously, the estimated DOAs become more accurate as the frequency is approaching the reference value, $4 \mathrm{GHz}$. This could be explained by noticing the decreasing steering vector transformation RMSE values shown in Fig. 4.

Fig. 5 shows six generated beam patterns corresponding to the six sub-bands. It is clear that the array beam patterns are maintained at $0 \mathrm{~dB}$ in the direction of the SOI and the nulls are placed correctly in the direction of the jammer. Fig. 6 shows the detailed 'zoomed' nulls' patterns. Since nulls' patterns are very sensitive to the errors in the estimated jammer DOA, the nulls' pattern values in the jammer direction become deeper as the frequency becomes closer to the reference frequency.

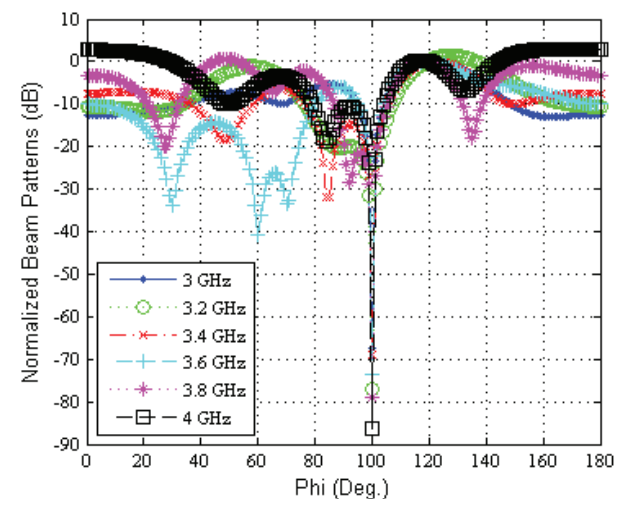

Fig. 5. Normalized array beam patterns.

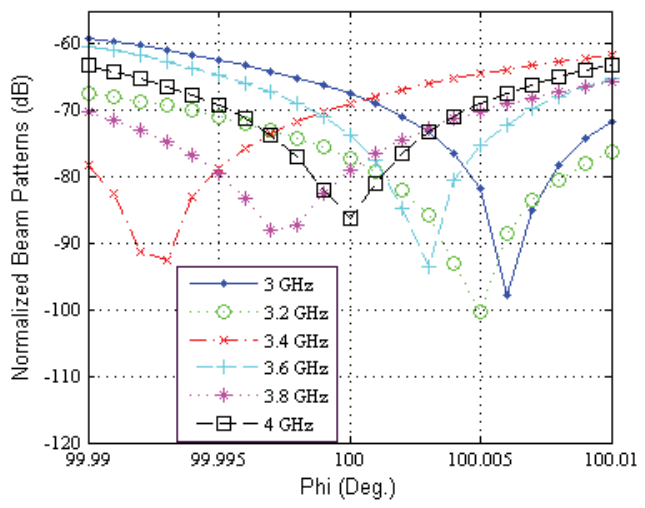

Fig. 6. Normalized detailed nulls' patterns.

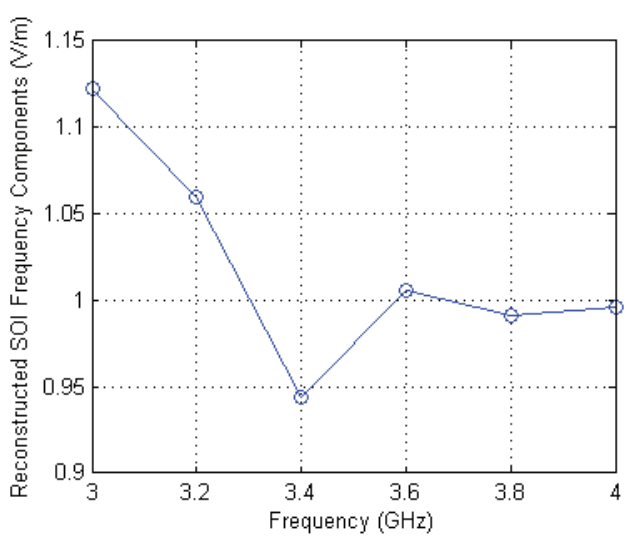

Fig. 7. Reconstructed SOI frequency components $(\mathrm{V} / \mathrm{m})$.

Fig. 7 shows the estimated magnitudes of the SOI frequency components using each corresponding beam pattern. Note that the estimation error is large at the lower frequencies and decreases as the reference frequency is approached. To improve the SOI frequency components' reconstruction accuracy, a hybrid reconstruction beam pattern is used. The hybrid pattern combines the most accurate estimated DOAs, i.e. DOAs estimated at the reference frequency, and the deepest nulls corresponding to the strongest estimated interferer frequency component since all the jammer frequency components magnitudes are equal, the already formed beam pattern corresponding to the highest frequency, $f_{o}$, is used as a single reconstruction beam pattern by which all the SOI frequency components' magnitudes are accurately estimated as shown in Fig. 8. 


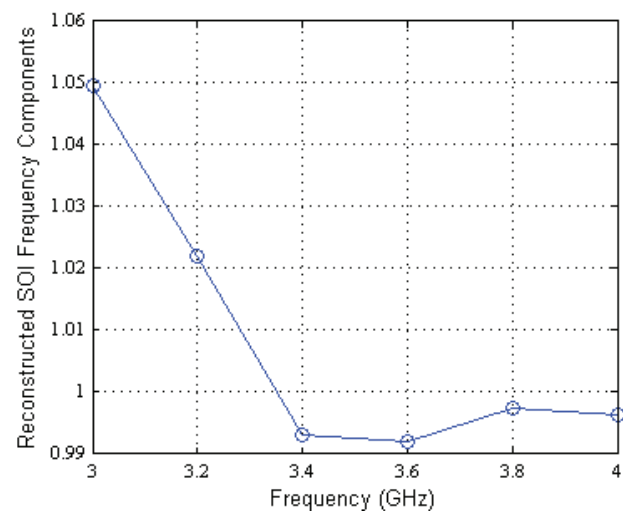

Fig. 8. Reconstructed SOI frequency components $(\mathrm{V} / \mathrm{m})$.

\subsection{Example 2: Varying Jammers' Frequency Components Magnitudes}

In the $2^{\text {nd }}$ example, all jammer frequency components' magnitudes are $60 \mathrm{~dB}$ over the corresponding SOI frequency components except the last two jammer frequency components, i.e. at $3.8 \mathrm{GHz}$ and $4 \mathrm{GHz}$, which are only $40 \mathrm{~dB}$ over the corresponding SOI frequency components' strengths. In this case, the beam pattern corresponding to the highest frequency sub-band has a null in the jammer direction but its depth is not sufficient to cancel the high jammer frequency components' strengths at the other subbands. Fig. 9 and Fig. 10 show the beam pattern corresponding to the highest frequency sub-band and the corresponding estimated SOI frequency components respectively. In Fig. 10, it is obvious that the estimations at the last two frequencies are acceptable but the estimations at the other frequencies, whereby the jammer frequency components' strengths are high, are not acceptable. These bad estimations could be improved by using a hybrid reconstruction beam pattern which combines the accurate DOA estimations at the reference frequency and the null depth corresponding to the strongest estimated jammer frequency component. The hybrid reconstruction pattern is shown in Fig. 11 and the corresponding estimated SOI frequency components are shown in Fig. 12. The improvement in the SOI frequency components estimations is clear as the null shown in Fig. 11 is deep enough to eliminate the strong jammer frequency components efficiently.

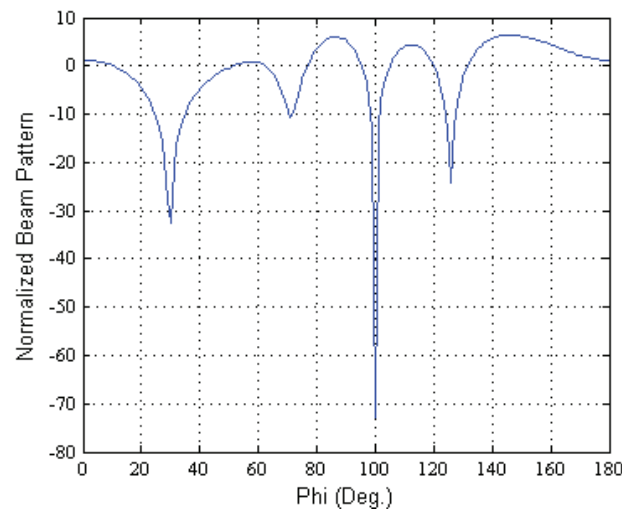

Fig. 9. Normalized beam pattern.

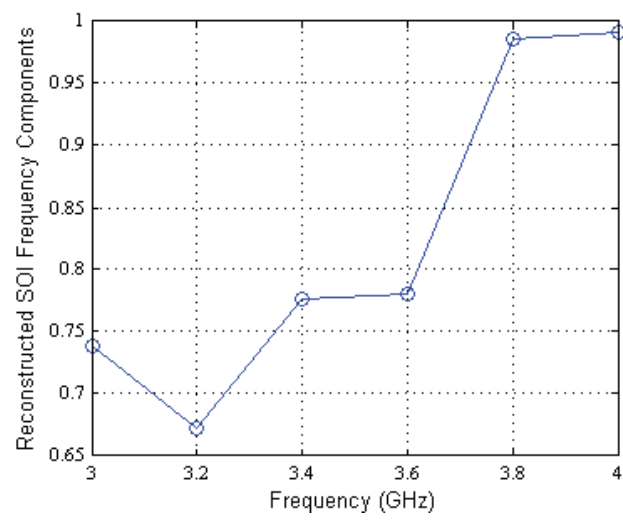

Fig. 10. Reconstructed SOI frequency components (V/m).

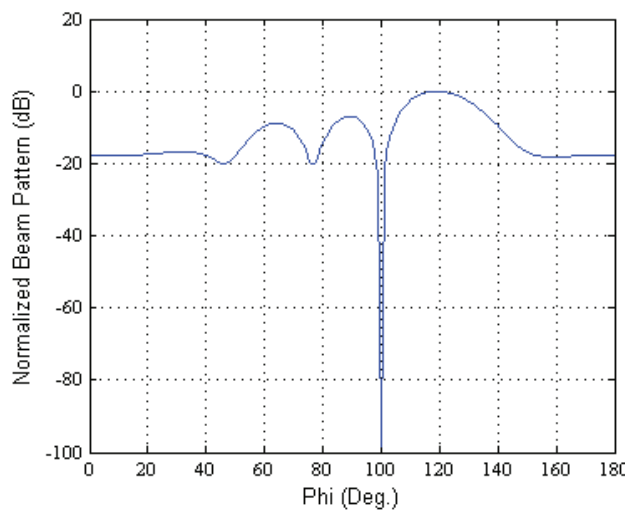

Fig. 11. Hybrid reconstruction beam pattern.

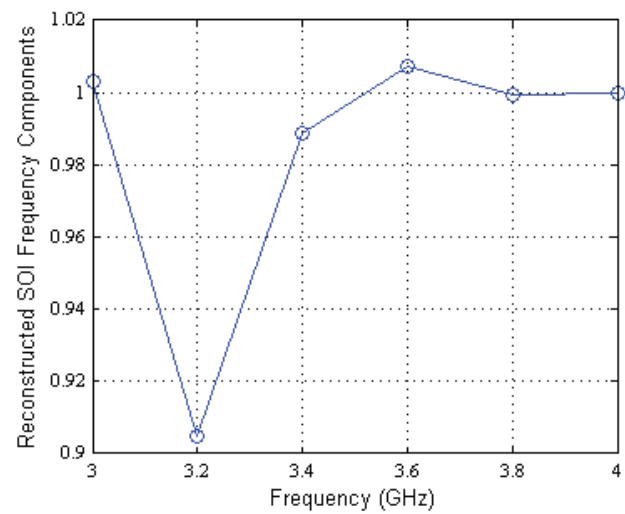

Fig. 12. Reconstructed SOI frequency components (V/m).

\section{Conclusion}

This paper presents a new wideband direct data domain genetic algorithm (WD ${ }^{3} \mathrm{GA}$ ) beamforming. The proposed beamformer is based on decomposing the received wideband signal into its narrow sub-bands using FFT. The beamformer is using a single hybrid reconstruction beam pattern which combines both sufficient deep nulls and accurate DOA estimations.

\section{References}

[1] ELKAMCHOUCHI, H., MOHAMED, D., ALI, W. D ${ }^{3}$ LS STAP approach on wideband signals using uniformly spaced real 
elements. International Journal of Computer Applications, 2011, vol. 22 , no. 4 , p. $42-47$. DOI: $10.5120 / 2568-3530$

[2] SVENDSEN, A., GUPTA, I. The effect of mutual coupling on the nulling performance of adaptive antennas. IEEE Antennas and Propagation Magazine, June 2012, vol. 54, no. 3, p. 17-38. DOI: 10.1109/MAP.2012.6293947

[3] ADVE, R., SARKAR, T. Compensation for the effects of mutual coupling on direct data domain adaptive algorithms. IEEE Transactions on Antennas and Propagation, January 2000, vol. 48, no. 1, p. $86-94$. DOI: $10.1109 / 8.827389$

[4] SARKAR, T., WICKS, M., SALAZAR-PALMA, M., BONNEAU, R. Smart Antennas. Wiley-IEEE Press, 2003.

[5] SHABAN, M., KISHK, S. Steering vector transformation technique for the design of wideband beamformer. In The Proceeding of $27^{\text {th }}$ National Radio Science Conference. Menouf (Egypt), 2010.

[6] LIU, W., WEISS, S. Wideband Beamforming Concepts and Techniques. Wiley (Wiley Series on Wireless Communication and Mobile Computing), 2010. 302 p. ISBN: 978-0-470-71392-1

[7] ELLATIF, W. Smart Antennas: Space Time Adaptive Processing Based on Direct Data Domain Least Squares Using Real Elements. Doctoral Dissertation. Electrical Engineering, Alexandria University, 2011.

[8] SARKAR, T., PEREIRA, O. Using the matrix pencil method to estimate the parameters of a sum of complex exponentials. IEEE Antennas and Propagation Magazine, May 1995, vol. 37, no. 1, p. 48-55. DOI: 10.1109/74.370583

[9] ELKAMCHOUCHI, H., HASSAN, M. Space time adaptive processing using real array elements based on direct data domain adaptive nulls genetic algorithm beam forming. In Proceedings of the International Conference on Electronics and Communication System. Coimbatore (India), 2014, vol. 2, p. 183-187. DOI: 10.1109/ECS.2014.6892563

[10] HASSAN, M. A Proposed Fault Identification Scheme in Systems Using Soft Computing Methodologies. M.Sc. Dissertation. Electrical Engineering, Alexandria University, 2008.

[11] Genetic Algorithm and Direct Search Tool Box User's Guide. The MathWorks, Inc., 2006.

[12] LIU, W., WEISS, S., HANZO, L. A generalized side lobe canceller employing two-dimensional frequency invariant filters.
IEEE Transactions on Antennas and Propagation, 2005, vol. 53, no. 7, p. 2339-2343. DOI: 10.1109/TAP.2005.850759

[13] LIU, W., WEISS, S. A new class of broad arrays with frequency invariant beam patterns. In Proceeding of International Conference on Acoustics, Speech, and Signal Processing ICASSP 2004. Montreal (Canada), 2004, vol. 2, p. 185-188. DOI: 10.1109/ICASSP.2004.1326225

[14] MOGHADDAM, P., AMINDAVAR, H. Direction of arrival estimation: a new approach. In Proceeding of Signal Processing Conference. Nordic (Sweden), 2000.

[15] MONTHIPPA, U., BIALKOWSKI, M. E. A wideband smart antenna employing spatial signal processing. Journal of Telecommunication and Information Technology, 2007, no. 1, p. $13-17$.

[16] REN, Y., HE, H., ZHANG, Y., ZHANG, K. An amplitude-only direct data domain least square algorithm of wideband signals based on the uniform circular array. In Proceeding of the $5^{\text {th }}$ International Conference on Wireless Communications, Networking and Mobile Computing (WiCom 2009). Beijing (China), 2009, p. 1-4. DOI: 10.1109/WICOM.2009.5302753

[17] MANI, V.V.; BOSE, R. Genetic algorithm based smart antenna design for UWB beamforming. In IEEE International Conference on Ultra-Wideband ICUWB 2007. Singapore, 2007, p. 442-446. DOI: 10.1109/ICUWB.2007.4380985

\section{About the Authors ...}

Hassan ELKAMCHOUCHI was born in Egypt, 1943. He received his Ph.D. degree in Communication Engineering in 1972 from Alexandria University. He is now a professor for Alexandria University, Egypt. His research interests include adaptive antennas, optimization algorithms, radars, electromagnetic theory, cryptography and digital communication.

Mohamed HASSAN was born in Egypt. He received his M.Sc. from Alexandria University in 2008. His research interests include adaptive antennas, optimization algorithms, radars, electromagnetic theory and modeling. 